\title{
Principales dificultades en el llenado de los certificados de defunción
}

\author{
Main difficulties found in filling the death certificates out
}

\author{
Dra. Odalys Rodríguez Martín,' Lic. Yanara Matos Valdivia,' Lic. Danoris \\ Anchia Alonso,' Dra. Miriela Betancourt Valladares" \\ ' Dirección Provincial de Salud. Departamento de Estadística. Ciego de Ávila, Cuba. \\ "Clínica Estomatológica del Instituto de Ciencias Médicas "Carlos J. Finlay". \\ Camagüey, Cuba.
}

\section{RESUMEN}

I ntroducción: uno de los pilares fundamentales en la toma de decisiones en salud pública es el conocimiento de las principales causas de muerte de la población. El Certificado Médico de Defunción es el modelo oficial y el registro primario del sistema de información estadística de mortalidad del país.

Objetivo: valorar el llenado de los certificados médicos de defunción a partir de los errores detectados.

Métodos: se realizó un estudio descriptivo longitudinal retrospectivo en la provincia de Ciego de Ávila en el año 2010. El universo estuvo constituido por 3334 certificados de defunción. La muestra quedó conformada por 324 certificados seleccionados por muestreo simple aleatorio por estar los certificados enumerados de forma consecutiva en la base de datos. Se diseñó una escala cualitativa validada por criterio de expertos para evaluar el correcto llenado del certificado.

Resultados: entre los errores más frecuentes se detectaron: omitir detalles o características de enfermedades como la enfermedad pulmonar obstructiva crónica en 22 de los certificados, no especificar la causa que conduce a bronconeumonía en personas de edad avanzada en 19, omitir el tiempo aproximado entre el inicio de la causa y la muerte en 17, omisión de la causa básica que lo llevó a la muerte en 12 y designar la causa básica como desconocida o muerte súbita en 6 , entre otros. Conclusiones: el llenado de los certificados médicos ha mejorado pero aún persisten errores que atentan contra la calidad de la información estadística de mortalidad. No se detectaron certificados de pésima calidad.

Palabras clave: Clasificación Internacional de Enfermedades, mortalidad, certificado médico de defunción, causa básica de muerte, secuencia. 


\section{ABSTRACT}

Introduction: one of the fundamental pillars of decision-making in the public health is the knowledge about the main causes of death in the population. The death certificate is the official form and the primary registry of the statistical information system of mortality nationwide.

Objective: to evaluate the filling out of the death certificates based on the detected errors.

Methods: a retrospective longitudinal and descriptive study was performed in Ciego de Avila province in 2010. The universe was 3334 death certificates, but the sample was finally made up of 324 selected by means of simple random sampling, since the certificates were consecutively numbered in the database. An expert criteria-validated qualitative scale was designed to evaluate the right filling out of the certificate.

Results: the most frequent errors were leaving details or characteristics of a disease(for example, chronic obstructive pulmonary disease) out in 22 certificates, do not specify the cause leading to bronchial pneumonia in the elderly in 19 of them, failing to mention the length of time elapsed from the onset of disease to the patient's death in 17 , omission of the basic cause leading to death in 12 certificates, and stating basic unknown cause or sudden death in 6 , among others.

Conclusions: filling medical certificates out has improved, but there are still errors hindering the quality of statistical information on mortality. No low quality death certificates are detected.

Key words: International Classification of Diseases, mortality, death certificate, basic cause.e, sequence.

\section{NTRODUCCI ÓN}

La implantación de la Clasificación Internacional de Enfermedades, Décima Revisión (CIE-10) en los países de la Región de las Américas, es parte importante de un proceso más amplio de revisión y mejoramiento de las estadísticas vitales según afirma la Organización Panamericana de la Salud (OPS). ${ }^{1}$

Uno de los pilares fundamentales en la toma de decisiones en salud pública es conocer cuáles son las principales causas de muerte de la población. El Certificado Médico de Defunción (CMD), es el modelo oficial y el registro primario del sistema de información estadística de mortalidad del país. ${ }^{1-3}$

El CMD es un documento de gran importancia legal de emisión obligatoria en todo fallecimiento, ya sea cualquier tipo de muerte (violenta, natural o sospechosa de criminalidad), o se trate de un individuo de nacionalidad cubana o extranjera. ${ }^{4}$

En todos los casos de muerte violenta o sospechosa de compromiso penal, se debe informar de inmediato a la unidad policial para que la autoridad correspondiente inicie el proceso de investigación, en estos casos el CMD debe ser confeccionado por médicos legistas y de ser posible por dos de ellos, plasmando los nombres de cada uno, el número de registro profesional y la firma. ${ }^{3}$ 
Las estadísticas de la mortalidad son fuentes tradicionales de información referente a la salud de las poblaciones y vienen siendo utilizadas en Epidemiología desde el siglo XVI. ${ }^{5}$

La certificación de las causas de muerte, es uno de los procederes médicos más antiguos. La certificación obligatoria de la defunción por el médico que atendió al paciente, fue una aspiración sostenida de la Junta Superior de Sanidad, que se fundó en Cuba en 1826 y fue legalizada y publicada en la prensa para general conocimiento el 22 de enero de $1843 .{ }^{6,7}$

El 20 de julio de 1901 entró en vigor el Sistema Internacional de Nomenclatura de Enfermedades y de Causas de Defunción, Clasificación de Bertillón. A partir de entonces, se han seguido las revisiones aproximadamente decenales de la $\mathrm{CIE}$ hasta la actualidad en que se realizó el cambio para la Décima Revisión en el 2002 y se puso en vigencia un sistema de captación de defunciones certificadas por médicos en todos los hospitales del Sistema Nacional de Salud, con uso de un certificado de defunción único. ${ }^{6,8}$.

Las estadísticas provenientes de la mortalidad registrada pueden verse alteradas en cualquier etapa de su producción: recolección de datos y llenado de formularios, codificación, procesamiento de datos y cómputo posterior. ${ }^{2}$

El reparo es una vía que utilizan los departamentos de registros médicos y estadística - los comité de la calidad hospitalaria en aquellos CMD que no especifiquen adecuadamente la enfermedad o lesión que inició la cadena de acontecimientos patológicos que condujeron directamente a la muerte. ${ }^{3}$

La causa consignada en el certificado de defunción, a menudo está incompleta o es de mala calidad, entre otras razones, por falta de capacitación en certificación y una comprensión insuficiente de los usos que se hacen de la información proporcionada en el certificado de defunción. ${ }^{9}$

En Cuba hay 3 tipos de certificados de defunción vigentes. EI CMD para ser usado en fallecidos de 28 días de edad y más, es el de mayor utilización en el país y donde se registran la mayor cantidad de errores en su llenado. ${ }^{3}$

Las estadísticas de mortalidad son una de las principales fuentes de información sobre salud y de ellas dependen, en gran medida, las políticas que asumen los países en esta materia. Contribuir a aumentar los conocimientos y habilidades en los estudiantes y profesionales para lograr un nivel superior en la certificación de las causas de muerte eleva la calidad de la certificación. ${ }^{10}$

La importancia y trascendencia de realizar una correcta certificación de la muerte ha sido señalada desde hace mucho tiempo por varios autores. Se han publicado varios trabajos referentes a la confección correcta de los certificados de defunción del adulto, persistiendo múltiples dificultades para realizar una secuencia lógica de las causas de muerte, lo cual los hace, en ocasiones, no trasmisores de toda la información correcta que de ellos se debe esperar. ${ }^{4,7,11}$

Habitualmente se reciben en el departamento de estadística provincial CMD con errores en el llenado surgiendo la necesidad de realizar reparos que pueden llevar a subregistros, razón que motiva a la realización de un estudio para valorar el llenado de los certificados médicos de defunción a partir de los errores detectados y que esto sirva de base para implementar cursos de posgrado para capacitar a los profesionales en este proceder y contribuir a mejorar la calidad y la confiabilidad de las estadísticas de mortalidad en la provincia. 


\section{MÉTODOS}

Se realizó un estudio descriptivo longitudinal retrospectivo en la provincia de Ciego de Ávila en el 2010. El universo estuvo constituido por 3334 certificados de defunción registrados en la base de datos del departamento provincial de estadística. Se utilizó el programa estadístico EPIDAT 3.1 para el cálculo del tamaño de muestra, se consideró una confiabilidad del $95 \%$, se utilizó una proporción del $63 \%$ de certificados llenados correctamente, ${ }^{4}$ y un máximo error admitido de $5 \%$. Para la selección de la muestra se utilizó el muestreo simple aleatorio por estar los certificados enumerados de forma consecutiva en la base de datos. La muestra quedó conformada por 324 certificados de defunción.

Se analizaron las variables edad, residencia, causa básica de muerte, unidad que llena el certificado e intervalo de muerte. Para realizar la valoración del correcto llenado del certificado de defunción se diseñó una escala cualitativa similar a la de otros paíse, ${ }^{2}$ adaptada a nuestro medio y validada por criterios de expertos. En la bibliografía consultada de estudios nacionales no se alude a escalas empleadas para evaluar el llenado de los certificados de defunción.

Se definieron los criterios de evaluación y se establecieron cuatro niveles:

- Llenado correcto: se consideró el certificado que no presenta errores en su llenado.

- Llenado admisible: se consideró la presencia de un error

- Llenado deficiente: se consideró la presencia de hasta dos errores.

- Llenado incorrecto: presencia de tres o más errores.

Toda la información necesaria fue recopilada en una base de datos confeccionada en el programa SPSS versión 15.0 para Windows y se resumió en frecuencias absolutas y porcentajes.

\section{RESULTADOS}

La calidad de las estadísticas de mortalidad depende de la calidad del llenado por el médico del Certificado Médico de Defunción.

De la muestra de certificados analizada, el grupo de edad que registró mayor número de defunciones fue el de 70 a 90 años.

De acuerdo a la unidad que certifica la muerte, el hospital "Ciego de Ávila" arrojó el mayor número de certificados con errores, seguido del hospital "Morón."

Entre los errores más frecuentes (tabla) se detectaron:

- omitir detalles o características de enfermedades como la enfermedad pulmonar obstructiva crónica (EPOC) en 22 de los certificados,

- no especificar las causas que llevaron a la bronconeumonía en personas de edad avanzada (debe aclararse si es hipostática o no) en 19 certificados; en este caso se 
hace reparo con el objetivo de buscar otras posibles causas que llevaron a la muerte,

- omitir el tiempo aproximado entre el inicio de la causa y la muerte, en 17, muy importante, porque puede modificar la causa de muerte,

- omisión de causa básica que lo llevó a la muerte, en 12 certificados,

- designar como causa básica, causa desconocida o muerte súbita, lo cual lleva un certificado con resultados de la necropsia y reparo, en 6 ocasiones,

- causa básica mal definida como única causa de muerte en 5 de los certificados y no existencia de la secuencia lógica de las enfermedades en 4 certificados.

Tabla. Distribución de Certificados Médicos de Defunción según errores detectados

\begin{tabular}{|l|c|}
\hline Errores detectados & $\begin{array}{c}\text { No. } \\
\text { de certificados* }\end{array}$ \\
\hline Omitir detalles o caracteristicas de enfermedades como el EPOC. & 22 \\
\hline $\begin{array}{l}\text { No especificar la cusa que lo lleva a bronconeumonía en personas } \\
\text { de edad avanzada, en este caso se hace reparo }\end{array}$ & 19 \\
\hline Omitir el tiempo aproximado entre el inicio de la causa y la muerte & 17 \\
\hline Omisión de causa básica que lo llevó a la muerte. & 12 \\
\hline Designar como causa básica: causa desconocida ó muerte súbita & 6 \\
\hline \begin{tabular}{l|c|} 
Causa básica mal definida como única causa \\
de muerte
\end{tabular} & 5 \\
\hline No existencia de secuencia lógica de las enfermedades & 4 \\
\hline Ordenamiento inverso de la secuencia & 1 \\
\hline $\begin{array}{l}\text { Que la causa básica no guarde relación con la causa intermedia ni } \\
\text { directa }\end{array}$ & 1 \\
\hline $\begin{array}{l}\text { Intervalo de evolución de la enfermedad no guarda relación con la } \\
\text { causa básica }\end{array}$ & 1 \\
\hline Complicaciones que se omiten de una enfermedad & 90 \\
\hline Causa básica mal definida & 1 \\
\hline Total & \\
\hline
\end{tabular}

* CMD con más de un error

Fuente: base de datos de CMD. Departamento Provincial de Estadística.

Del total de los certificados estudiados, $241(74,4 \%)$ tenían un llenado correcto, un llenado admisible, $76(23,5 \%)$ y solamente $7(2,1 \%)$ tenían un llenado deficiente. No se detectaron certificados con llenado incorrecto.

\section{DISCUSI ÓN}

El Sistema de Información Estadística de Mortalidad en Cuba, provee información estadística oportuna y confiable. ${ }^{3}$ 
Países como Perú arrojan resultados diferentes y existen problemas de subregistro. Evaluación realizada por la Organización Mundial de la Salud (OMS) incluye a este país dentro del grupo de países con baja calidad en el sistema de registro de mortalidad. $^{2}$

En Bolivia en 1999 el subregistro de mortalidad se estimó en 63 \%. Además del subregistro, otro problema en los datos de mortalidad es el referido a la calidad de la certificación de la defunción. ${ }^{8}$

El mayor número de defunciones son registradas en edades avanzadas, siendo frecuente encontrar la bronconeumonía como única causa de muerte, en tal caso se solicita reparo buscando otras posibles causas que lo llevaron al enfermo a la bronconeumonía, lo cual no quiere decir que esta enfermedad no pueda ser una causa básica y sea tabulada como tal, pero en estas edades, en la mayoría de los fallecidos, viene siendo una complicación, por tal motivo el pensamiento médico juega un papel fundamental. Debe consignarse la causa básica que realmente dio inició a la cadena de acontecimientos que condujeron directamente a la muerte.

Es evidente que en los hospitales acontece el mayor número de fallecidos, razón por la cual emiten mayor número de certificado con errores.

Estudios internacionales y nacionales han puesto en evidencia dificultades en la confección del CMD donde se señalan errores que concuerdan con los encontrados en este estudio. ${ }^{4,12}$

Debe destacarse que consignar una sola causa de muerte en el certificado puede denotar poco conocimiento de la evolución del paciente y de sus antecedentes patológicos personales, en ocasiones se indica la enfermedad que ocasionó directamente la muerte y se omiten aquellas que le dieron origen o se excluyen enfermedades que pudieran ser consecuencias o complicaciones de la afección inicial. $^{3}$

En un estudio similar realizado en Perú donde se emplea una escala similar en la evaluación de los certificados, de 287 certificados de defunción revisados, solo 97 $(33,8 \%)$ se consideran como buenos y $128(44,6 \%)$ de llenado pésimo o de mala calidad. $^{2}$

Publicaciones nacionales informan que en 53 diabéticos fallecidos, solo en 19 está consignada la diabetes como la causa básica o principal de la muerte; en un estudio sobre la mortalidad por trastornos metabólicos se aprecia una elaboración inadecuada del certificado de defunción por los médicos de atención primaria. ${ }^{11,7}$

La OMS, en investigaciones realizadas en Ciudad del Cabo, revela información útil en el $75 \%$ de los certificados de defunción, el $60 \%$ de los fallecidos con una sola causa certificada y solo el $55 \%$ de los formularios codificados con precisión. Tales discrepancias son debidas a la codificación de la causa inmediata, no la subyacente. ${ }^{12}$

Aunque los datos fueron obtenidos de la base de datos, y los CMD no fueron revisados directamente, sí se conoció que se dificulta el vaciamiento de estos por los problemas de redacción durante el llenado. Los médicos al expedir los Certificados de Defunción están en la obligación de redactarlo con una letra legible y sin usar siglas o abreviaturas no autorizadas; se escribirá con tinta azul o negra y no podrá tener enmiendas ni tachaduras. ${ }^{3}$ 
El trabajo desplegado en la provincia para darle solución al problema del llenado en los CMD, bajo el liderazgo de la Dirección Provincial de Salud y el trabajo del personal de estadística, se dirigió a elevar su calidad.

El llenado de certificados médicos ha mejorado aunque aún persisten errores que deben ser solucionados para mejorar la calidad de la información estadística de mortalidad.

\section{Agradecimientos}

A Aida Isabel Sánchez Rodríguez y Sonia Lucia Ravelo Moreno, Técnico Básico en Estadísticas de Salud y Técnico Medio en Gestión de la Información en Salud, respectivamente, por su colaboración en la realización de este trabajo.

\section{REFERENCI AS BI BLI OGRÁFICAS}

1. Organización Panamericana de la Salud. Implementación de la Clasificación estadística Internacional de enfermedades y problemas relacionados con la salud, décima revisión (CIE-10). Boletín Epidemiol [Internet] 1997 [citado 3 oct 2010]; 18(1). Disponible en: http://www.paho.org/spanish/sha/epibul_9598/bs971cie.htm

2. Soto Caceres V. Calidad del llenado de los certificados de defunción en un hospital público de Chiclayo. Rev Perú Med Exp Salud Pública [Internet]. 2008 [citado 5 Ene 2011];25(3). Disponible en :

http://scielo.sld.cu/scielo.php?script=sci arttext\&pid=S0864-

$03192003000100003 \&$ lng $=p t \& n r m=i s o \& t \operatorname{lng}=e s$

3. Zacca Peña E, Martinez Morales MA. ¿Como llenar un certificado médico de defunción? Reflexiones e instrucciones en torno a un llenado correcto [Internet]. 2010 [citado 3 Oct 2010]. Disponible en:

http://www. uvs.sld.cu/profesores/supercursos/plonearticlemultipage. 2006-05$\underline{18.7979805252 /}$

4. Jorge Miguez AN. La importancia de llenar bien el certificado de defunción. Medisur [Internet]. 2009 [citado 10 Nov 2010]; 7(6). Disponible en:

http://scielo.sld.cu/scielo.php?script $=$ sci_arttext\&pid=S1727-

897X2009000600001\&lng=es\&nrm=iso

5. Carvalho de Oliveira Pedrosa LD, Wanick Sarinho S, de Albuquerque Rocha Ordonha M. Causas básicas de las muertes neonatales en Brasil: conocer para prevenir. Rev Cubana Pediatr [Internet]. 2006 [citado 3 Ene 2011]; 78(4) Disponible en: http://scielo.sld.cu/scielo.php?script=sci_arttext\&pid=S003475312006000400008\&lng=es

6. Ríos Massabot NE, Fernández Viera RM, Jorge Pérez ER. Los Registros Médicos en Cuba. Rev Cubana Salud Pública [Internet]. 2005 [citado 5 Ene 2011];31(4).

Disponible en: http://scielo.sld.cu/pdf/rcsp/v31n4/spu13405.pdf

7. Barreiro Ramos H, Barreiro Peñaranda A, Fernández Viera E, Marrero Martín O. Certificado médico de defunción del adulto. Rev Cubana Med Gen Integr [Internet]. 2004 [citado 10 Nov 2010];20(4). Disponible en: 
http://scielo.sld.cu/scielo.php?script=sci_arttext\&pid=S0864$\underline{21252004000400011 \& \operatorname{lng}=e s \& n r m=i s o \& t \operatorname{lng}=e s}$

8. Organización Panamericana de la Salud. Análisis Coyuntural de la Mortalidad en Bolivia. Boletín Epidemiol [Internet]. 2002 [citado 3 Ene 2011];23(2). Disponible en: http://www.paho.org/spanish/sha/be_v23n2-mortalidadbolivial.htm

9. Organización Panamericana de la Salud. Sobre la estimación de tasas de mortalidad para países de la Región de las Américas. Boletín Epidemiol. [Internet]. 2003 [citado 3 Oct 2010]; 24(4). Disponible en:

http://www. paho.org/spanish/dd/ais/be_v24n4-mortalidad.htm

10. Cabrera Carrillo G, Barreiro Ramos H, Barreiro Peñaranda A. Impacto de curso de posgrado en la atención primaria de salud. Educ Med Super [Internet]. 2006 [citado 10 Nov 2011];20(3). Disponible en:

http://scielo.sld.cu/scielo.php?script=sci_arttext\&pid=S0864-

$21412006000300003 \& \operatorname{lng}=\mathrm{es}$

11. Barreiro Ramos H, Barreiro Peñaranda A, Fernández Viera E, Marrero Martín O. Lo esencial del nuevo certificado médico de defunción del adulto. Rev Cubana Med Gen Integr [Internet]. 2002 [citado 10 Nov 2010]; 18(4). Disponible en:

http://scielo.sld.cu/scielo.php?script=sci_arttext\&pid=S0864-

$\underline{21252002000400011 \& \operatorname{lng}=\mathrm{es}}$

12. Bradshaw D, Groenewald P, Bourne DE, Mahomed H, Nojilana B, Daniela J, et.al. Estadísticas sobre las causas de defunción: utilidad para la salud pública a nivel local en Ciudad del Cabo. Boletín de la Organización Mundial de la Salud [Internet]. 2008 [citado 10 Nov 2010];84(3). Disponible en: http://www. who.int/bulletin/volumes/84/3/bradshaw0306abstract/es/

Recibido: 18 de mayo de 2011.

Aprobado: 2 de marzo de 2012.

Odalys Rodríguez Martín. Dirección Provincial de Salud. Departamento de Estadística. Ciego de Ávila, Cuba. Correo electrónico: odalysedgar@gmail.com 